\title{
NEGATIVE PARTITION RELATIONS FOR ULTRAFILTERS ON UNCOUNTABLE CARDINALS
}

\author{
AKI KANAMORI AND ALAN D. TAYLOR
}

\begin{abstract}
Assuming GCH, we prove that if $\kappa$ is a successor cardinal and $U$ is a uniform ultrafilter on $\kappa$, then $U \nrightarrow(U, 3)^{2}$. The case $\kappa=\omega_{1}$ is an old result of Hajnal. Our proof makes use of several known results concerning nonregular, weakly normal and indecomposable ultrafilters, as well as some negative partition relations for uncountable ordinals.
\end{abstract}

0. Introduction. An ultrafilter $U$ on $\kappa$ is said to be uniform if every set in $U$ has cardinality $\kappa$. We consider the question of when an infinite cardinal $\kappa$ can carry a uniform ultrafilter $U$ satisfying the following partition relation.

Definition 0.1. $U \rightarrow(U, 3)^{2}$ iff whenever $A \in U$ and $f:[A]^{2} \rightarrow 2$, either there is a set $B \in U$ such that $f^{\prime \prime}[B]^{2}=\{0\}$, or else there is a set $X \in[A]^{3}$ so that $f^{\prime \prime}[X]^{2}=\{1\}$.

Graph theoretically, $U \rightarrow(U, 3)^{2}$ is the assertion that every triangle-free graph with vertex set in $U$ has an independent subgraph whose vertex set is also in $U$. The arrow notation is an analogue of the usual disjunctive partition relation for ordinals of Erdös, Hajnal, and Rado; other variants of this notation, such as $U \rightarrow(U, k)^{2}$ or $U \rightarrow(W, \omega)^{2}$, will also be used.

Ultrafilters on $\kappa=\omega$ satisfying $U \rightarrow(U, 3)^{2}$ have been fairly extensively studied (see [BT]), and if $\kappa$ is a measurable cardinal and $U$ is a normal ultrafilter on $\kappa$, then it follows a fortiori from Rowbottom's well-known partition theorem [R, 4.8] that $U \rightarrow(U, 3)^{2}$. But, for arbitrary $\kappa>\omega$, much less is known, and this is our main concern. In $\$ 1$ we show that if $U$ is a uniform ultrafilter on an uncountable cardinal $\kappa$ and $U \rightarrow(U, 3)^{2}$, then $U$ is a separating ultrafilter (as defined in [KT]). It follows from this that if $\mathrm{CH}$ holds, then $U$ is nonregular and there is a measurable cardinal in an inner model. In $\$ 2$ we show that if there is a uniform ultrafilter $U$ on $\kappa$ satisfying $U \rightarrow(U, 3)^{2}$ and $\operatorname{cf}(\kappa)>2^{\omega}$, then there is a weakly normal ultrafilter $V$ on $\kappa$ that also satisfies $V \rightarrow(V, 3)^{2}$. The indecomposability of ultrafilters satisfying $U \rightarrow(U, 3)^{2}$ is discussed in $\S 3$ and these results are then used to demonstrate that if the GCH holds, then no successor cardinal $\kappa$ can carry an ultrafilter $U$ satisfying $U \rightarrow(U, 3)^{2}$. The special case where $\kappa=\omega_{1}$ is an unpublished result of Hajnal (see $\S 3)$. We conclude in $\$ 4$ with two examples of countably incomplete uniform ultrafilters $U$ satisfying $U \rightarrow(U, 3)^{2}$; one is on a regular cardinal and the other is on a singular cardinal.

Received by the editors March 29, 1983.

1980 Mathematics Subject Classification. Primary 03E05; Secondary 03E35, 03 E55. 
1. Separating and nonregular ultrafilters. A uniform ultrafilter $U$ on $\kappa$ is said to be a separating ultrafilter iff whenever $f, g$ are defined on $\kappa$ and $f(\alpha) \neq g(\alpha)$ for $U$-almost every $\alpha$, then there exists a set $B \in U$ such that $f^{\prime \prime} B \cap g^{\prime \prime} B=0$. The relevance of this notion to our present considerations is shown by the following; it is a special case of a more general result in [T].

THEOREM 1.1. If $U$ is a uniform ultrafilter on $\kappa$ and $U \rightarrow(U, 3)^{2}$, then $U$ is a separating ultrafilter.

Proof. Suppose that $A \in U$ and $f, g: A \rightarrow \kappa$ are such that for every $\alpha \in A$ we have $f(\alpha) \neq g(\alpha)$. Define $h:[A]^{2} \rightarrow 2$ so that if $\alpha<\beta$ then

$$
h(\{\alpha, \beta\})=0 \text { iff } f(\alpha) \neq g(\beta) .
$$

It is easy to see that if $X \in[A]^{3}$, then $h^{\prime \prime}[X]^{2} \neq\{1\}$. Hence, there is a set $Y \in U$ such that $Y \subseteq A$ and $h^{\prime \prime}[Y]^{2}=\{0\}$. If we now repeat the above procedure starting with $Y$ in place of $A$ and with the roles of $f$ and $g$ reversed, then we arrive at a set $Z \in U$ such that $Z \subseteq Y$ and $f^{\prime \prime} Z \cap g^{\prime \prime} Z=0$.

It should be noted that the converse of Theorem 1.1 fails. For example, assuming $\mathrm{CH}$ it is possible to construct an ultrafilter $U$ on $\omega$ so that $U$ is separating but $U \nrightarrow(U, 3)^{2}$. In fact, even the existence of a separating ultrafilter on $\kappa$ need not entail the existence of a uniform ultrafilter $U$ on $\kappa$ satisfying $U \rightarrow(U, 3)^{2}$; e.g. for $\kappa=\omega_{1}$ the former is consistent with $\mathrm{CH}$ (unpublished), while the latter is inconsistent with $\mathrm{CH}$ by Hajnal's observation (see $\S 3$ ).

Recall that a uniform ultrafilter $U$ on $\kappa$ is said to be $(\lambda, \mu)$-regular iff there are $\mu$ sets in $U$, any $\lambda$ of which have empty intersection. $U$ is said to be regular if it is $(\omega, \kappa)$-regular. The following results are now immediate consequences of Theorem 1.1 and known properties of separating ultrafilters (see [KT, Theorems 2.1, 2.5, and 3.2], where the results are established with " $U \rightarrow(U, 3)^{2}$ " replaced by "separating").

COROLlaRY 1.2. Suppose that $U$ is a uniform ultrafilter on $\kappa$ and $U \rightarrow(U, 3)^{2}$. Then

(a) $U$ is non- $\left(\omega, 2^{\omega}\right)$-regular, and

(b) if $\kappa=\lambda^{+}$, where $\lambda$ is an uncountable strong limit cardinal, then $U$ is not $(\gamma, \kappa)$-regular for any $\gamma<\lambda$.

COROLlaRY 1.3. Suppose that $U$ is a uniform ultrafilter on $\kappa>\omega, U \rightarrow(U, 3)^{2}$, and either

(a) $\mathrm{CH}$ holds, or

(b) $\kappa>2^{\kappa_{0}}$ and $\kappa^{<\kappa}=\kappa$.

Then there is an inner model with a measurable cardinal.

2. $P$-points and weakly normal ultrafilters. A uniform ultrafilter $U$ on $\kappa$ is said to be a $P$-point if for every $f: \kappa \rightarrow \kappa$ there is a set $X \in U$ such that either $f$ is $<\kappa$ to 1 on $X$ or $\left|f^{\prime \prime} X\right|<\kappa$. If $U$ is an ultrafilter on $\kappa$ and $D$ is an ultrafilter on $\lambda$, then we write $D \leqslant{ }_{\mathrm{RK}} U$ iff there is a function $f: \kappa \rightarrow \lambda$ so that $X \in D$ iff $f^{-1}(X) \in U$; in this case we say that $D$ lies below $U$ in the Rudin-Keisler ordering and we write $D=f_{*}(U)$. Our interest in this section is with the special case in which $U$ and $D$ are 
both ultrafilters on $\kappa$. It is easy to see that if $U \rightarrow(U, 3)^{2}$ and $D \leqslant_{\mathrm{RK}} U$, then $D \rightarrow(D, 3)^{2}$.

It turns out that an ultrafilter satisfying $U \rightarrow(U, 3)^{2}$ need not be a $P$-point. For example, as pointed out in [BT], if $U$ is the product of distinct normal ultrafilters on the measurable cardinal $\kappa$, then $U \rightarrow(U, 3)^{2}$, but $U$ is not a $P$-point. We do, however, have the following

THEOREM 2.1. Suppose that $U$ is a uniform ultrafilter on the infinite cardinal $\kappa$ and $U \rightarrow(U, 3)^{2}$. Then there is a P-point ultrafilter $D$ on $\kappa$ so that $D \rightarrow(D, 3)^{2}$ and $D \leqslant \mathrm{RK}_{\mathrm{R}} U$.

Proof. If $U$ itself is a $P$-point then we are done, so suppose that $f: \kappa \rightarrow \kappa$ shows that $U$ is not a $P$-point and let $D=f_{*}(U)$. Notice that $D$ is a uniform ultrafilter on $\kappa$ and so we need only show that $D$ is a $P$-point. Assume for contradiction that $g$ : $\kappa \rightarrow \kappa$ shows that $D$ is not a $P$-point. Define $F:[\kappa]^{2} \rightarrow \kappa$ as follows. If $\alpha<\beta<\kappa$, then set

$$
F(\{\alpha, \beta\})=1 \quad \text { iff } \quad g(f(\alpha))<f(\alpha)<g(f(\beta))<\alpha<f(\beta)<\beta .
$$

It is easy to see that if $Y \subseteq \kappa$ and $|Y|=3$, then $F^{\prime \prime}[Y]^{2} \neq\{1\}$. Since $U \rightarrow(U, 3)^{2}$, there is a set $X \in U$ such that $F^{\prime \prime}[X]^{2}=\{0\}$.

Let $X_{1}=\left\{\gamma<\kappa: f^{-1}(\{\gamma\})\right.$ is unbounded in $\left.\kappa\right\}$ and let $X_{2}=\left\{\gamma<\kappa: g^{-1}(\{\gamma\})\right.$ $\cap X_{1}$ is unbounded in $\left.\kappa\right\}$. Notice that $f^{-1}\left(X_{1}\right) \in U$ since $f$ is $<\kappa$ to 1 on $\kappa-f^{-1}\left(X_{1}\right)$, and $g^{-1}\left(X_{2}\right) \in D$ since $g$ is $<\kappa$ to 1 on $X_{1}-g^{-1}\left(X_{2}\right)$. Thus $X_{3} \in U$, where

$$
X_{3}=X \cap f^{-1}\left(X_{1}\right) \cap f^{-1}\left(g^{-1}\left(X_{2}\right)\right) .
$$

Choose $\alpha_{1} \in X_{2}$ and choose $\alpha_{2} \in X_{1}$ such that $\alpha_{2}>\alpha_{1}$ and $g\left(\alpha_{2}\right)=\alpha_{1}$. Choose $\beta_{1} \in X_{2}$ so that $\beta_{1}>\alpha_{2}$ and choose $\alpha_{3} \in X_{3}$ so that $\alpha_{3}>\beta_{1}$ and $f\left(\alpha_{3}\right)=\alpha_{2}$. Choose $\beta_{2}>\alpha_{3}$ so that $\beta_{2} \in X_{1}$ and $g\left(\beta_{2}\right)=\beta_{1}$. Finally, choose $\beta_{3} \in X_{3}$ so that $\beta_{3}>\beta_{2}$ and $f\left(\beta_{3}\right)=\beta_{2}$. It is now easy to see that $F\left(\left\{\alpha_{3}, \beta_{3}\right\}\right)=1$, contradicting the fact that $F^{\prime \prime}[X]^{2}=\{0\}$.

The reader familiar with partition relations for ordinals will recognize the above proof as being based on the obvious extension of Specker's example [S] showing that $\omega^{3} \rightarrow\left(\omega^{3}, 3\right)^{2}$.

Recall that a uniform ultrafilter $D$ on $\kappa$ is said to be weakly normal iff whenever $\{\alpha<\kappa: f(\alpha)<\alpha\} \in D$, there is a set $X \in D$ such that $\left|f^{\prime \prime} X\right|<\kappa$. The following lemma is from [Ka]; it is stated there only for the special case in which $\kappa$ is a regular cardinal, but the argument clearly yields the more general result.

LEMMA 2.2. Suppose that $\operatorname{cf}(\kappa) \geqslant \omega_{1}$ and $D$ is a uniform ultrafilter on $\kappa$ that is both non- $(\omega, \mathrm{cf}(\kappa))$-regular and a P-point. Then there exists a weakly normal ultrafilter $V$ on $\kappa$ such that $V \leqslant_{\mathrm{RK}} D$.

The following is now an immediate consequence of Corollary 1.2(a), Theorem 2.1 and Lemma 2.2 . 
THEOREM 2.3. Suppose that $\operatorname{cf}(\kappa) \geqslant 2^{\omega}$ and $U$ is a uniform ultrafilter on $\kappa$ satisfying $U \rightarrow(U, 3)^{2}$. Then there exists a weakly normal ultrafilter $V$ on $\kappa$ so that $V \rightarrow(V, 3)^{2}$ and $V \leqslant_{\mathrm{RK}} U$.

3. Indecomposable ultrafilters and successor cardinals. Our goal in this section is to prove that if the GCH holds, then no successor cardinal $\kappa$ can carry a uniform ultrafilter $U$ satisfying $U \rightarrow(V, 3)^{2}$. The first result along these lines seems to be the following observation of Hajnal.

THEOREM 3.1 (HAJNAL). If $\mathrm{cf}(\kappa)>\omega$ and $U$ is a countably incomplete uniform ultrafilter on $\kappa$ satisfying $U \rightarrow(U, 3)^{2}$, then $\kappa \cdot \omega \rightarrow(\kappa \cdot \omega, 3)^{2}$.

COROllaRy 3.2 (HAJNAL). If $C H$ holds and $U$ is a uniform ultrafilter on $\omega_{1}$, then $U \nrightarrow(U, 3)^{2}$.

Hajnal proves Theorem 3.1 by noting that every countably incomplete uniform ultrafilter on $\kappa$ is naturally isomorphic to an ultrafilter $U$ on the index set $\kappa \cdot \omega$ with the property that if $X \in U$, then $X$ has order-type $\kappa \cdot \omega$. The corollary then follows from the $\lambda=\omega$ case of Hajnal's theorem:

Theorem 3.3 (HaJnal [EH]). If $2^{\lambda}=\lambda^{+}$, then $\lambda^{+} \cdot \lambda \nrightarrow\left(\lambda^{+} \cdot \lambda, 3\right)^{2}$.

In one sense, the optimal way to extend Corollary 3.2 to other successor cardinals would be to extend Hajnal's negative partition relation by proving that if $2^{\lambda}=\lambda^{+}$, then $\lambda^{+} \cdot \omega \nrightarrow\left(\lambda^{+} \cdot \omega, 3\right)^{2}$. This route, however, appears to be an extremely difficult one. Moreover, there are inherent limitations to this approach, since it is consistent that the converse of Theorem 3.1 fails. That is, an old theorem of Erdös and Hajnal [EH] (generalized by Baumgartner and Larson [L]) asserts that if $\kappa$ is weakly compact, then $\kappa \cdot \omega \rightarrow(\kappa \cdot \omega, 3)^{2}$, but it is clear from the results of $\S 1$ that if $V=L$, $\kappa$ is weakly compact and $U$ is a uniform ultrafilter on $\kappa$, then $U \nrightarrow(U, 3)^{2}$.

Given this, we instead will obtain the desired generalization of Corollary 3.2 by directly strengthening Theorem 3.1 via the following well-known property of ultrafilters: if $U$ is a uniform ultrafilter on $\kappa$, then $U$ is said to be $\lambda$-decomposable iff there is a uniform ultrafilter $V$ on $\lambda$ such that $V \leqslant_{\mathrm{RK}} U$. Notice that if $U$ is countably incomplete, then $U$ is $\omega$-decomposable. Thus, Theorem 3.1 becomes a special case of the following

THEOREM 3.4. If $\lambda<\operatorname{cf}(\kappa)$ and $\kappa \cdot \lambda \nrightarrow(\kappa \cdot \lambda, 3)^{2}$, then any uniform ultrafilter $U$ on $\kappa$ satisfying $U \rightarrow(U, 3)^{2}$ must be $\lambda$-indecomposable.

Proof. In order to prove that $U$ is $\lambda$-indecomposable, it suffices to show that if $f$ : $\kappa \rightarrow \lambda$, then there is a set $X \in U$ such that $\left|f^{\prime \prime} X\right|<\lambda$. Without loss of generality, assume that $U$ is on the index set $\lambda \times \kappa$ and that $f(x, y)=x$ for every $(x, y) \in \lambda \times \kappa$. Let $F:[\lambda \times \kappa]^{2} \rightarrow 2$ be a counterexample showing that $\kappa \cdot \lambda \nrightarrow(\kappa \cdot \lambda, 3)^{2}$. Since $U \rightarrow(U, 3)^{2}$, there exists a set $Y \in U$ such that $F^{\prime \prime}[Y]^{2}=\{0\}$. Notice that if

$$
Z=\{\alpha<\lambda:|\{\beta<\kappa:(\alpha, \beta) \in Y\}|=\kappa\}
$$


then $|Z|<\lambda$. Let $X=Y \cap(Z \times \kappa)$. Since $Y-X$ is a union of at most $\lambda$ sets each of size less than $\kappa$, and $\operatorname{cf}(\kappa)>\lambda$, we have that $|Y-X|<\kappa$ and so $X \in U$ because $U$ is a uniform ultrafilter on $\kappa$. But $f^{\prime \prime} X=Z$ and $|Z|<\lambda$, and so this is the desired result.

As an immediate consequence of Theorems 3.3 and 3.4, we obtain the following generalization of Corollary 3.2.

COROLlaRY 3.5. If $2^{\lambda}=\lambda^{+}$and $U$ is a uniform ultrafilter on $\lambda^{+}$satisfying $U \rightarrow(U, 3)^{2}$, then $U$ is $\lambda$-indecomposable.

If we now combine Corollary 3.5 with the nonregularity results of $\S 1$, the weak normality results of $\$ 2$, and several well-known properties of indecomposable ultrafilters, we obtain the main result of this section.

THEOREM 3.6. If $2^{\lambda}=\lambda^{+}$, where $\lambda$ is either regular or a singular strong limit cardinal, and if $U$ is a uniform ultrafilter on $\lambda^{+}$, then $U \nrightarrow(U, 3)^{2}$.

Proof. Assume for contradiction that $U \rightarrow(U, 3)^{2}$, and thus, by Corollary 3.5 , that $U$ is $\lambda$-indecomposable. By a result of Kunen and Prikry [KP], if $\lambda$ is regular, then any uniform ultrafilter on $\lambda^{+}$is $\lambda$-decomposable. Thus, we need only consider the case where $\lambda$ is a singular strong limit cardinal. By Theorem 2.3, we lose no generality in assuming that $U$ is also weakly normal.

With the given cardinal hypotheses, we can conclude from Theorem 12 of $[\mathbf{P r}]$ that $U$ is either $\lambda$-decomposable or else $\beta$-decomposable for arbitrarily large $\beta<\lambda$. Since we are assuming the former fails, we have

$$
U \text { is } \beta \text {-decomposable for arbitrarily large } \beta<\lambda \text {. }
$$

Now, a theorem of Kunen and Prikry [KP] states that if $\lambda$ is singular and $U$ is a uniform ultrafilter on $\lambda^{+}$, then either $U$ is $\left(\gamma, \lambda^{+}\right)$-regular for some regular $\gamma<\lambda$ or else $U$ is $\operatorname{cf}(\lambda)$-decomposable. Since Corollary 1.2(b) rules out the former alternative, we have

$U$ is $\operatorname{cf}(\lambda)$-decomposable.

Finally, by Proposition 1 of [Pr], (*) together with (**) entail that $U$ is $\lambda$-decomposable, and this is the desired contradiction.

COROLlARY $3.7(\mathrm{GCH})$. If $U$ is a uniform ultrafilter on a successor cardinal, then $U \nrightarrow(U, 3)^{2}$.

COROLLARY $3.8(\mathrm{GCH})$. If there is a uniform ultrafilter $U$ on a regular uncountable cardinal satisfying $U \rightarrow(U, 3)^{2}$, then there is such an ultrafilter on an inaccessible cardinal $\kappa$ which is also $\beta$-indecomposable for every regular uncountable $\beta<\kappa$.

Proof. Suppose that $U$ on $\lambda$ is as hypothesized. Let $\kappa \leqslant \lambda$ be the least regular uncountable cardinal such that $U$ is $\kappa$-decomposable. If $V \leqslant_{\mathrm{RK}} U$ is uniform on $\kappa$ then $\kappa$ is inaccessible by Corollary 3.7, and $V$ has the desired properties.

4. Examples. We conclude with two examples of countably incomplete ultrafilters satisfying $U \rightarrow(U, 3)^{2}$, one on a singular cardinal and one on a regular cardinal. The 
proofs in this section are somewhat tedious but relatively straightforward; hence they are omitted.

THEOREM 4.1. Suppose that $U$ is a uniform ultrafilter on $\omega$ satisfying $U \rightarrow(U, 3)^{2}$, $\left\langle\kappa_{n}: n \in \omega\right\rangle$ is an increasing sequence of measurable cardinals, and $U_{n}$ is a uniform $\kappa_{n}$-complete ultrafilter on $\kappa_{n}$ satisfying $U_{n} \rightarrow\left(U_{n}, 3\right)^{2}$ for each $n \in \omega$. Let $\lambda=\sup \kappa_{n}$, and define $D$ by

$$
X \in D \quad \text { iff } \quad X \subseteq \lambda \quad \text { and } \quad\left\{n \in \omega: X \cap \kappa_{n} \in U_{n}\right\} \in U
$$

Then $D$ is a uniform countably incomplete ultrafilter on the singular cardinal $\lambda$ satisfying $D \rightarrow(D, 3)^{2}$.

It is also not hard to verify that if $U \rightarrow(U, \omega)^{2}$ and $U_{n} \rightarrow\left(U_{n}, \omega\right)^{2}$ for every $n \in \omega$, then the resulting ultrafilter $D$ on $\lambda$ will also satisfy $D \rightarrow(D, \omega)^{2}$. Thus, a singular cardinal can carry a countably incomplete uniform ultrafilter $D$ satisfying $D \rightarrow(D, \omega)^{2}$. The following observation shows that a regular (uncountable) cardinal cannot.

THEOREM 4.2. Suppose that $\kappa$ is a regular cardinal and $U$ is a uniform ultrafilter on $\kappa$ satisfying $U \rightarrow(U, \omega)^{2}$. Then $U$ is $\kappa$-complete (and so $\kappa$, if uncountable, is a measurable cardinal ).

Proof. We can verify that $U$ is $\kappa$-complete by establishing that whenever $\lambda<\kappa$ and $\kappa=\bigcup\left\{X_{\alpha}: \alpha<\lambda\right\}$ is a partition of $\kappa$ into $\lambda$ pieces, then there is a $\gamma<\lambda$ so that $\bigcup\left\{X_{\alpha}: \alpha<\gamma\right\} \in U$. To do this, define $f:[\kappa]^{2} \rightarrow 2$ so that if $\xi<\eta$, then $f(\{\xi, \eta\})=0$ iff $\xi \in X_{\alpha}, \eta \in X_{\beta}$ and $\alpha \leqslant \beta$. Notice that if $f^{\prime \prime}[X]^{2}=\{1\}$, then $|X|<\omega$ or else we would have an infinite descending sequence of ordinals. So, by hypothesis, there is a set $A \in U$ such that $f^{\prime \prime}[A]^{2}=\{0\}$. By the regularity of $\kappa$ and the uniformity of $U$, there must be a $\delta<\lambda$ such that $\left|A \cap X_{\delta}\right|=\kappa$. But then, $A \cap X_{\beta}=0$ for $\delta<\beta<\lambda$ by the definition of $f$, as $A$ has order-type $\kappa$. Thus $\bigcup\left\{X_{\alpha}: \alpha<\delta+1\right\} \in U$, and we are done.

In contrast to Theorem 4.2, our final example shows that a regular uncountable cardinal can carry a uniform countably incomplete ultrafilter $D$ satisfying $D \rightarrow$ $(D, 3)^{2}$. By mimicking some of the arguments in [BT], a slightly stronger result than what we give is possible, but only at the expense of considerably complicating the proof. The notation $V \rightarrow(V)_{2}^{2}$ means that if $X \in V$ and $f:[X]^{2} \rightarrow 2$, then there is a set $Y \in V$ so that $f$ is constant on $[Y]^{2}$.

THEOREM 4.3. Suppose that $U$ is a uniform ultrafilter on $\omega$ satisfying $U \rightarrow(U, 3)^{2}$ and $V$ is a $\kappa$-complete ultrafilter on the (uncountable) measurable cardinal $\kappa$ satisfying $V \rightarrow(V)_{2}^{2}$. Let $D$ be the product ultrafilter $U \times V$; i.e., $X \in D$ iff $X \subseteq \omega \times \kappa$ and $\{n<\omega:\{\alpha<\kappa:(n, \alpha) \in X\} \in V\} \in U$. Then $D$ is a uniform countably incomplete ultrafilter on (a set of size) $\kappa$ and $D \rightarrow(D, 3)^{2}$.

\section{REFERENCES}

[B] J. Baumgartner, Partition relations for uncountable cardinals, Israel J. Math. 21 (1975), 296-307.

[BT] J. Baumgartner and A. Taylor, Partition theorems and ultrafilters, Trans. Amer. Math. Soc. 241 (1978), 283-309. 
[EH] P. Erdös and A. Hajnal, Ordinary partition relations for ordinal numbers, Period. Math. Hungar. 1 (1971), 171-185.

[Ka] A. Kanamori, Weakly normal filters and irregular ultrafilters, Trans. Amer. Math. Soc. 220 (1976), 393-399.

[KT] A. Kanamori and A. Taylor, Separating ultrafilters on uncountable cardinals, Israel J. Math. (to appear).

[KP] K. Kunen and K. Prikry, On descendingly incomplete ultrafilters, J. Symbolic Logic 36 (1971), 650-652.

[L] J. Larson, Partition theorems for certain ordinals products, preprint.

[Pr] K. Prikry, On descendingly complete ultrafilters, Cambridge Summer School in Math. Logic (A.R.D. Mathias and H. Rogers, Jr., eds.), Lecture Notes in Math., vol. 337, Springer-Verlag, Berlin and New York, 1973, pp. 459-488.

[R] F. Rowbottom, Some strong axioms of infinity incompatible with the axiom of constructibility, Ann. Math. Logic 3 (1971), 1-44.

[S] E. Specker, Teilmengen von Mengen mit Relationen, Comment. Math. Helv. 31 (1957), 302-314.

[T] A. Taylor, Separating collections (in preparation).

Department of Mathematics, Boston University, Boston, MassachusetTs 02215

Department of Mathematics, Union College, Schenectady, New York 12308 